\title{
Elevated pretreatment serum lactate dehydrogenase level predicts inferior overall survival and disease-free survival after resection of thymic carcinoma
}

\author{
Qianwen Liu ${ }^{1,2,3,4 *}$, Xiayu Fu ${ }^{1,2,3,4 *}$, Xiaodong $\mathrm{Su}^{1,2,3,4 *}$, Xin Wang ${ }^{1,2,3,4}$, Yijun Zhang ${ }^{2,3,4,5}$, Hong Yang ${ }^{1,2,3,4}$, Yi \\ $\mathrm{Hu}^{1,2,3,4}$, Jing Wen ${ }^{1,2,3,4}$, Jianhua Fu $\mathbf{u}^{1,2,3,4}$ \\ ${ }^{1}$ Department of Thoracic Surgery, ${ }^{2}$ State Key Laboratory of Oncology in South China, ${ }^{3}$ Guangdong Esophageal Cancer Institute, ${ }^{4}$ Collaborative \\ Innovation Center of Cancer Medicine, ${ }^{5}$ Department of Pathology, Sun Yat-sen University Cancer Center, Guangzhou 510060, China \\ Contributions: (I) Conception and design: J Wen, J Fu; (II) Administrative support: Q Liu, J Fu; (III) Provision of study materials or patients: All \\ authors; (IV) Collection and assembly of data: X Fu, X Su, Y Zhang; (V) Data analysis and interpretation: Q Liu, X Fu, X Su, X Wang, Y Zhang; (VI) \\ Manuscript writing: All authors; (VII) Final approval of manuscript: All authors. \\ *These authors contributed equally to this work. \\ Correspondence to: Jing Wen. Sun Yat-sen University Cancer Center, 651 Dongfeng Road East, Guangzhou 510060, China. Email: wenjing@sysucc.org.cn; \\ Jianhua Fu. Department of Thoracic Surgery, Sun Yat-sen University Cancer Center, 651 Dongfeng Road East, Guangzhou 510060, China. \\ Email: fujhsysucc@yahoo.com; fujh@sysucc.org.cn.
}

Background: The prognostic significance of serum lactate dehydrogenase (LDH) level in thymic carcinoma (TC) remains unclear. Therefore, we evaluated the role of pretreatment serum LDH level in the prognosis for TC in this study.

Methods: Sixty consecutive surgical patients were analyzed in this study with pathologic confirmed TC in Sun Yat-sen University Cancer Center from June 1996 to June 2014.

Results: The cut-off value of LDH was 210.50 IU/L. In both univariate analysis and multivariable analysis, only pretreatment serum LDH level $(\mathrm{P}=0.027)$ and pathological Masaoka stage $(\mathrm{P}=0.041)$ were associated with overall survival (OS). In univariate analysis, pretreatment serum LDH level, tumor size, postoperative radiotherapy (PORT) and pathological Masaoka stage were associated with disease-free survival (DFS) (all $\mathrm{P}<0.050)$. Multivariable analysis showed that LDH level $(\mathrm{P}=0.001)$, PORT $(\mathrm{P}=0.001)$ and pathological Masaoka stage $(\mathrm{P}=0.038)$ were independently prognostic factors of DFS. This study also revealed that male patients and larger tumor size had a significantly higher rate of elevated pretreatment serum LDH level than in the other groups.

Conclusions: In conclusion, pretreatment serum LDH level was an independent prognosis factor of OS and DFS for patients with TC.

Keywords: Lactate dehydrogenase (LDH); thymic carcinoma (TC); overall survival (OS); disease-free survival (DFS)

Submitted Mar 19, 2017. Accepted for publication Jul 17, 2017.

doi: $10.21037 /$ jtd.2017.10.86

View this article at: http://dx.doi.org/10.21037/jtd.2017.10.86

\section{Introduction}

Thymic carcinoma (TC) is relatively rare thymic neoplasms (1), accounts for $10 \%$ to $20 \%$ of thymic epithelial tumors (2-4). Surgical resection is the mainstay treatment for TC (5). To date, some studies on treatment modality and prognostic factors after surgery of TC have been published (2,4,6-18). However, these issues have not been well clarified.

TC is an aggressive neoplasm of the anterior mediastinum, and is hard to be found until the presence of symptoms such as chest pain, shortness of breath, cough, and hoarseness, caused by the pressure effect of the large tumor burden to 
surrounding mediastinal tissues. The large tumor burden might result in elevated serum lactate dehydrogenase (LDH) levels (19). Elevated serum LDH levels were considered as a negative prognostic indicator for many solid tumors, including small-cell lung cancer (20), nasopharyngeal carcinoma (21), germ cell tumors (22), and others. As for TC, no study on serum LDH has been reported.

Therefore, in the present study, medical records of TC patients underwent surgical treatment were retrospectively analyzed. Pretreatment serum LDH levels were evaluated, particularly with respect to their role in prognostic significance of TC.

\section{Methods}

\section{Study population}

Sixty consecutive surgical patients were analyzed in this study with pathologic confirmed TC in Sun Yat-sen University Cancer Center from June 1996 to June 2014. The inclusion criteria were: (I) histologically confirmed TC and (II) surgical resection. Patients who only underwent surgical biopsy were excluded. Patients' clinical and demographic information (gender, age, symptom, smoking history, alcohol abusing history, family history of any cancer, tumor size, surgical approach, locoregional invasion, completeness of resection, PORT, pre/postoperative chemotherapy, histology, pathological Masaoka stage, recurrence, and prognosis) was retrospectively reviewed. Histologic subtypes were determined by the 2004 World Health Organization (WHO) histologic classification. The stage of TC was classified based on the Masaoka staging system. A waiver of informed consent was requested, and the approval was obtained from independent ethics committees at Sun Yat-Sen University Cancer Center (number of the ethic approval: B2017-041).

\section{Laboratory measurement of $L D H$}

All patients analyzed in this study had serum LDH levels measured at admission for primary treatment. Briefly, $5 \mathrm{~mL}$ of peripheral blood was collected, centrifuged at $3,000 \mathrm{~g}$ for $5 \mathrm{~min}$ at room temperature, and then the plasma sample was carefully separated and transferred to polypropylene tubes. The samples were stored at $-20^{\circ} \mathrm{C}$ until further processing. Levels of LDH were tested by Hitachi Automatic Analyzer 7600-020 (Hitachi High-Technologies, Tokyo, Japan), and the coefficient of variance of a $\mathrm{LDH}$ measurement was
$<5.0 \%$. Normal serum LDH enzyme activities ranged from 109 to $245 \mathrm{IU} / \mathrm{L}$.

\section{Treatment}

All patients underwent surgery. Thoracotomy and sternotomy were performed in 43 patients and 17 patients, respectively. Involvement of adjacent organs was confirmed during operation. Pericardium invasion occurred in 24 patients, followed by invasion of the lung in 23 and invasion of the great vessels in 9. Forty-one patients were treated with postoperative radiotherapy (PORT), 4 patients received neoadjuvant chemotherapy, and 22 patients received postoperative chemotherapy.

\section{Follow-up}

A follow-up examination was generally carried out every 3 months for the first 2 years and twice a year thereafter. The regular follow-up assessment included physical examination, blood chemistry analysis, tumor markers, computed tomography scan, and ultrasonography. However, examinations were performed sooner if the patient had specific symptoms. The last censoring date for survival was June 2015. Two (3.3\%) patients were lost to follow-up. The mean follow-up of patients was 73.3 months ranging from 8.8 to 204.8 months.

\section{Statistical analysis}

Overall survival (OS) was measured from the date of surgery to the date of death or last follow-up. Disease-free survival (DFS) was determined as the time interval between date of surgery and the date when recurrence was noted or the date of last follow-up without recurrence for patients with complete resection. The survival curves were generated using the Kaplan-Meier method. Survival differences between the two groups were tested using the log-rank test. Prognostic factors for survival were calculated with Cox regression univariate and multivariate analysis. Any variables reaching $\mathrm{P}=0.05$ were introduced in multivariate analysis. The optimal cut-off value of $\mathrm{LDH}$ was determined using $\mathrm{X}$-tile software (http://www.giuspen.com/x-tile/) (23). The chi-square test or Fisher's exact test was applied as appropriate for the comparison of backgrounds of patients with a low level of $\mathrm{LDH}$ and patients with a high level of LDH. Two-sided $\mathrm{P}<0.050$ was considered statistically significant. Statistical analysis was performed by SPSS version 16.0 (SPSS, 
Chicago, IL, USA) statistical software.

\section{Results}

\section{Patient characteristics}

The median age was 50 years (range, $13-83$ years). Among 60 patients, 47 achieved $\mathrm{R} 0$ resection. The most frequent histological subtypes were squamous cell carcinoma $(n=37$, $61.67 \%)$, followed by lymphoepithelioma-like carcinoma $(\mathrm{n}=11,18.33 \%)$, and other tumors $(\mathrm{n}=12,20.00 \%)$. The pathological stage distribution according to the Masaoka system was as follows: stage I, 17 (28.33\%); stage IIa, 3 (5.00\%); stage IIb, 6 (10.00\%); stage III, 24 (40.00\%); stage IVa, 4 (6.67\%); and stage IVb, 6 (10.00\%). The clinical and histologic characteristics of these patients are shown in Table 1.

\section{Pretreatment serum LDH levels}

The median serum LDH levels for the entire cohort was $179.00 \mathrm{IU} / \mathrm{L}$, with values ranging from 109.00 to $685.10 \mathrm{IU} / \mathrm{L}$. The optimal cut-off value of LDH determined by X-tile software was $210.50 \mathrm{IU} / \mathrm{L}$. Of the 60 patients with TC, 39 (65.00\%) had pretreatment serum LDH levels less than or equal to $210.50 \mathrm{IU} / \mathrm{L}$, and $21(35.00 \%)$ had serum LDH levels higher than $210.50 \mathrm{IU} / \mathrm{L}$.

\section{Survival and prognostic analysis}

The overall 1-, 3-, 5-, and 10 -year survival rate were $88.2 \%$, $75.4 \%, 66.1 \%$, and $62.8 \%$, respectively. The $1-, 3-, 5-$, and 10 -year DFS rate were $78.7 \%, 66.8 \%, 58.5 \%$, and $42.3 \%$, respectively. The 1-, and 3-year survival after recurrence were $53.8 \%$, and $37.8 \%$, respectively. In both univariate analysis and multivariable analysis, only pretreatment serum $\mathrm{LDH}$ level (hazard ratio $=2.749, \mathrm{P}=0.027$ ) (Figure $1 A$ ) and pathological Masaoka stage (hazard ratio $=2.931, \mathrm{P}=0.041$ ) were associated with OS (Table 2). In univariate analysis, pretreatment serum LDH level $(\mathrm{P}=0.008)$ (Figure $1 B)$, tumor size $(\mathrm{P}=0.034)$, PORT $(\mathrm{P}=0.029)$ and pathological Masaoka stage $(\mathrm{P}=0.005)$ were associated with DFS. Multivariate analysis was also performed to adjust for various prognostic factors. Consistent with the univariate analysis results, pretreatment serum LDH level (hazard ratio $=4.587, \mathrm{P}=0.001$ ) was found to be an independent prognostic factor for DFS. PORT (hazard ratio $=5.752$, $\mathrm{P}=0.001$ ) and pathological Masaoka stage (hazard ratio
$=2.841, \mathrm{P}=0.038)$ were also identified as independent prognostic factors for DFS (Table 3).

\section{Association between pretreatment serum LDH level and other clinical and histologic characteristics}

The elevated pretreatment serum LDH level was associated with male patients $(\mathrm{P}=0.034)$ and larger tumor size $(\mathrm{P}=0.042)$. However, it was not associated with age, symptom, smoking history, alcohol abusing history, family history of any cancer, surgical approach, completeness of resection, PORT, pre/postoperative thermotherapy, histology, or pathological Masaoka stage (Table 1).

\section{Treatment}

The treatment modalities are listed in Table 4. All patients underwent surgical resection. Forty-seven patients received complete resection and 13 received incomplete resection. Forty-one patients were treated with PORT (prescribed dose ranged from 46 to 70 Gy). Twenty-three patients received chemotherapy (4 patients received neoadjuvant chemotherapy, 8 patients received postoperative chemotherapy, 14 patients received chemoradiotherapy). Among 47 patients with complete resection, 25 patients developed recurrence. After recurrence, 13 patients did not receive any treatment. Two patients received chemoradiotherapy. Nine patients received chemotherapy. One patient underwent surgical resection (Table 5).

\section{Discussion}

Our study demonstrated several important findings: (I) pretreatment serum LDH was a strong predictor of OS and DFS for TC patients treated with surgical resection; (II) PORT could prolong DFS but not OS; (III) male patients and larger tumor size were associated with elevated pretreatment serum LDH level.

TC is a rare neoplasm that is biologically and morphologically distinct from thymoma (24). According to WHO classification (25), TC was classified as a subtype of thymic epithelial neoplasms, including large cell carcinoma, small cell carcinoma, thymic neuroendocrine carcinoma and some other infrequent entities (3). Due to the limited cases, clinicopathologic features and treatment modalities have not yet been established. Masaoka staging (26) is considered as the most widely used prognostic tool for thymic epithelial neoplasms, however, the reliability is still controversial. 
Table 1 Clinicopathological characteristics

\begin{tabular}{|c|c|c|c|}
\hline Variables & $\mathrm{LDH} \leq 210.50 \mathrm{U} / \mathrm{L}$ & $\mathrm{LDH}>210.50 \mathrm{U} / \mathrm{L}$ & $P$ value \\
\hline Gender & & & 0.034 \\
\hline Male & 23 & 18 & \\
\hline Age (year) & & & 0.639 \\
\hline$\leq 60$ & 30 & 15 & \\
\hline Symptom & & & 0.349 \\
\hline Yes & 27 & 12 & \\
\hline No & 12 & 9 & \\
\hline Smoking history & & & 0.978 \\
\hline Yes & 3 & 3 & \\
\hline No & 36 & 18 & \\
\hline Family history of any cancer & & & $0.423^{*}$ \\
\hline Yes & 6 & 1 & \\
\hline No & 33 & 20 & \\
\hline Tumor size $(\mathrm{cm})$ & & & 0.042 \\
\hline$\leq 5$ & 13 & 2 & \\
\hline$>5$ & 26 & 19 & \\
\hline Surgical approach & & & 0.528 \\
\hline Yes & 26 & 15 & \\
\hline No & 13 & 6 & \\
\hline Pre/postoperative chemotherapy & & & 0.101 \\
\hline Yes & 12 & 11 & \\
\hline No & 27 & 10 & \\
\hline Histology & & & 0.805 \\
\hline Squamous cell carcinoma & 23 & 14 & \\
\hline Lymphoepithelioma-like carcinoma & 8 & 3 & \\
\hline Other & 8 & 4 & \\
\hline Pathological Masaoka stage & & & 0.251 \\
\hline $\mathrm{I}+\mathrm{II}$ & 19 & 7 & \\
\hline III+IV & 20 & 14 & \\
\hline
\end{tabular}

*, Fisher's exact test. LDH, lactate dehydrogenase. 

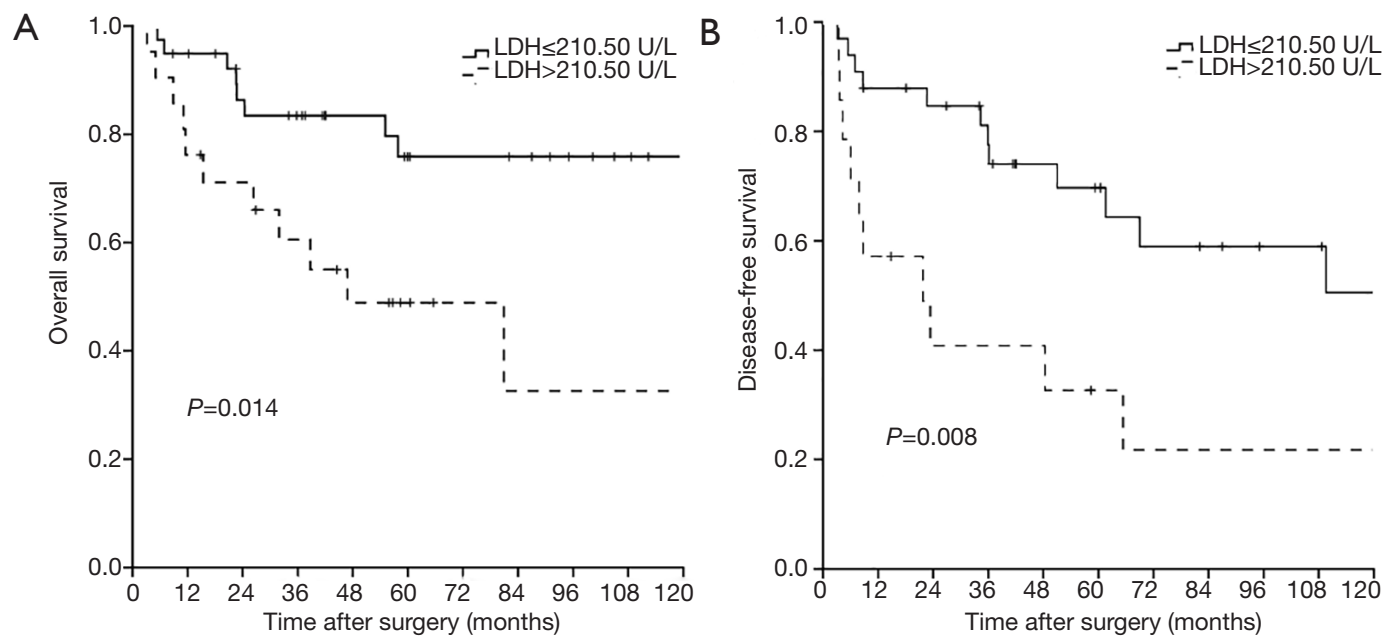

Figure 1 Kaplan-Meier estimation of overall survival and disease-free survival according to LDH levels. Compared to the lower subset (LDH $\leq 210.50 \mathrm{U} / \mathrm{L})$, elevated serum LDH level (>210.50 U/L) had an inferior overall survival (A) and disease-free survival (B). LDH, lactate dehydrogenase.

Table 2 Univariate and multivariate prognostic factors analysis for overall survival

\begin{tabular}{|c|c|c|c|c|c|c|}
\hline Variables & \multicolumn{3}{|c|}{ Univariate } & \multicolumn{3}{|c|}{ Multivariate } \\
\hline Gender & & & 0.188 & & & - \\
\hline Male & 1 & Reference & & - & - & \\
\hline Female & 0.486 & $0.162-1.458$ & & - & - & \\
\hline$\leq 60$ & 1 & Reference & & - & - & \\
\hline$>60$ & 0.276 & $0.064-1.192$ & & - & - & \\
\hline Symptom & & & 0.104 & & & - \\
\hline Yes & 1 & Reference & & - & - & \\
\hline No & 0.893 & $0.356-2.241$ & & - & - & \\
\hline Alcohol abusing history & & & 0.338 & & & - \\
\hline Yes & 1 & Reference & & - & - & \\
\hline No & 0.388 & $0.052-2.899$ & & - & - & \\
\hline Family history of any cancer & & & 0.462 & & & - \\
\hline Yes & 1 & Reference & & - & - & \\
\hline No & 0.575 & $0.129-2.555$ & & - & - & \\
\hline
\end{tabular}

Table 2 (continued) 
Table 2 (continued)

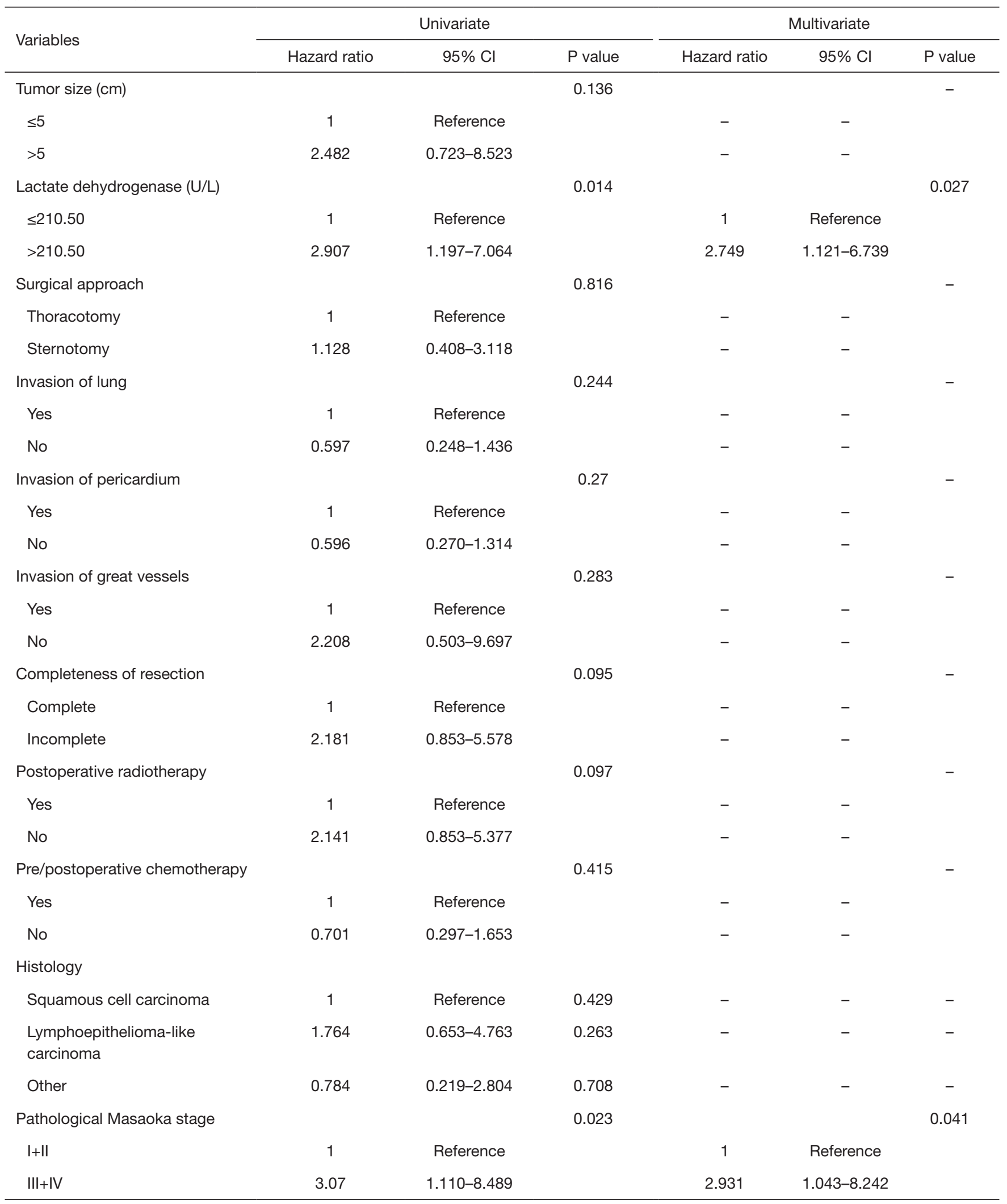


Table 3 Univariate and multivariate prognostic factors analysis for disease-free survival

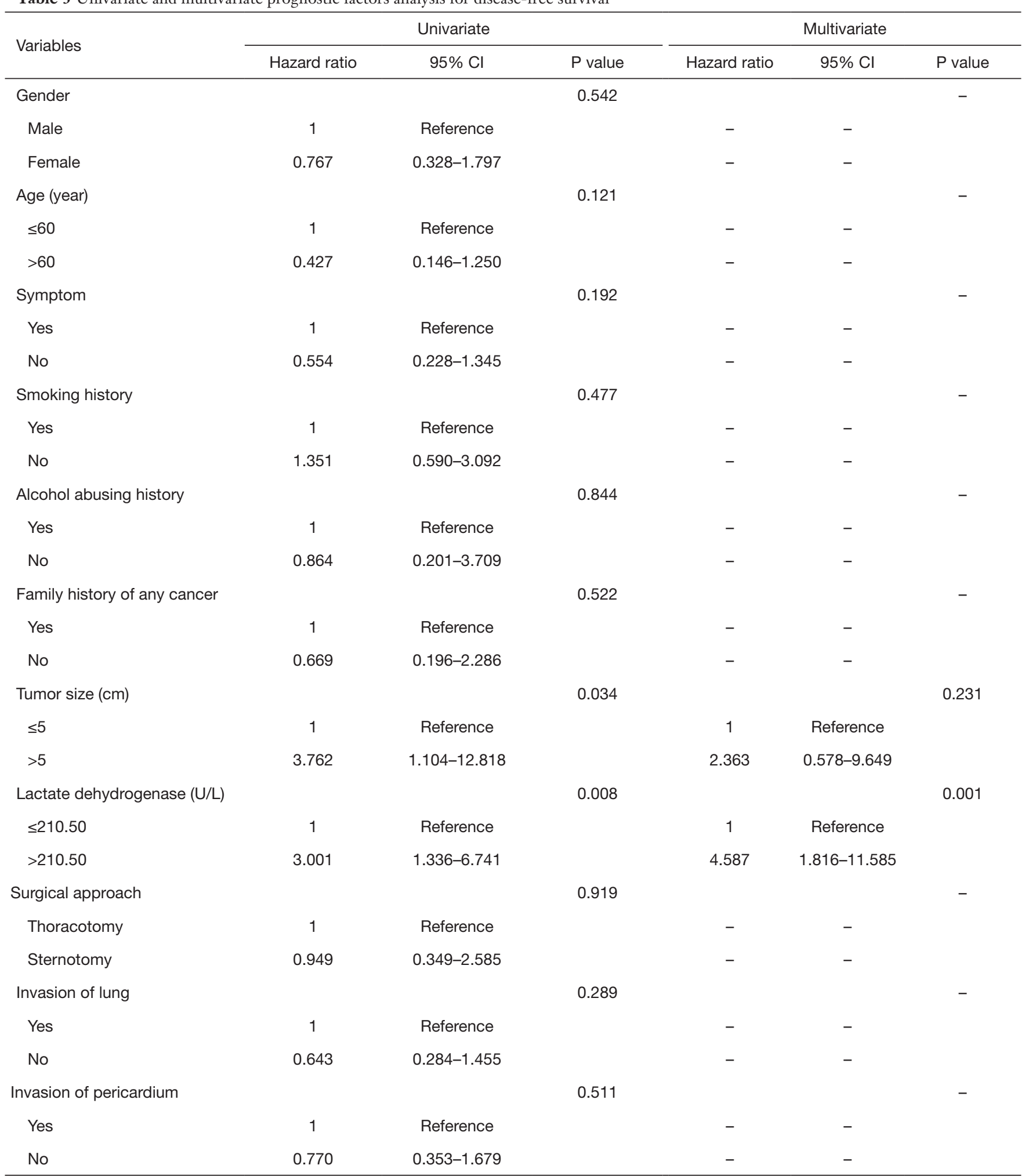

Table 3 (continued) 
Table 3 (continued)

\begin{tabular}{|c|c|c|c|c|c|c|}
\hline Variables & \multicolumn{3}{|c|}{ Univariate } & \multicolumn{3}{|c|}{ Multivariate } \\
\hline Invasion of great vessels & & & 0.982 & & & - \\
\hline Yes & 1 & Reference & & - & - & \\
\hline No & 0.987 & $0.328-2.973$ & & - & - & \\
\hline Yes & 1 & Reference & & 1 & Reference & \\
\hline No & 2.686 & $1.104-6.534$ & & 5.752 & $2.065-16.023$ & \\
\hline Pre/postoperative chemotherapy & & & 0.259 & & & - \\
\hline Yes & 1 & Reference & & - & - & \\
\hline Squamous cell carcinoma & 1 & Reference & 0.244 & - & - & - \\
\hline $\begin{array}{l}\text { Lymphoepithelioma-like } \\
\text { carcinoma }\end{array}$ & 1.886 & $0.732-4.859$ & 0.189 & - & - & - \\
\hline Other & 2.104 & $0.756-5.853$ & 0.154 & - & - & - \\
\hline Pathological Masaoka stage & & & 0.005 & & & 0.038 \\
\hline $\mathrm{I}+\mathrm{ll}$ & 1 & Reference & & 1 & Reference & \\
\hline III+IV & 3.326 & $1.438-7.693$ & & 2.841 & $1.060-7.615$ & \\
\hline
\end{tabular}

Table 4 Treatment modality

\begin{tabular}{lc}
\hline Variables & $\mathrm{n}(\%)$ \\
\hline S alone & $11(18.3)$ \\
$\mathrm{S}+\mathrm{RT}$ & $26(43.3)$ \\
$\mathrm{S}+\mathrm{CT}$ & $6(10.0)$ \\
$\mathrm{S}+\mathrm{CRT}$ & $13(21.7)$ \\
$\mathrm{CT}+\mathrm{S}+\mathrm{CT}$ & $2(3.3)$ \\
$\mathrm{CT}+\mathrm{S}+\mathrm{RT}$ & $1(1.7)$ \\
$\mathrm{CT}+\mathrm{S}+\mathrm{CRT}$ & $1(1.7)$ \\
\hline
\end{tabular}

$\mathrm{S}$, surgery; RT, radiotherapy; CT, chemotherapy; CRT, chemoradiotherapy.

Compared to thymoma, TC possesses more aggressive biological behavior, with a higher tendency for early relapse (27). Mixture of thymoma and TC may largely result in the stage migration, especially for TC $(28,29)$. Therefore, it is necessary to identify novel prognostic factors.

Our study demonstrated that pretreatment serum LDH
Table 5 Treatment after recurrence

\begin{tabular}{lc}
\hline Treatment & $\mathrm{n}(\%)$ \\
\hline CRT & $2(8.0)$ \\
CT & $9(36.0)$ \\
S + CT & $1(4.0)$ \\
No treatment & $13(52.0)$ \\
\hline
\end{tabular}

was strongly associated with OS and DFS. LDH is a crucial enzyme in the interconversion of pyruvate to lactate in anaerobic glycolysis (30). Commonly, the enzyme is stored in many different types of cell and maintains a relatively low serological level. LDH is abundant in some organs, such as heart, liver, kidney and muscle. When these organs are affected by malignancies, inflammation and toxicity, LDH releases from the cracked cell, which finally results in the elevation of serum LDH level $(31,32)$. Rapid progression of malignancies leads to low-oxygen conditions in tumor microenvironment (33), for adapting to these conditions, 
internal anaerobic metabolism of cancer cells is highly activated (34), therefore, LDH acts as a biomarker for tumor burden, and reflects tumor progression and tumor burden (35). In resent, several studies on cancer related elevation of LDH have been reported, and prognostic significance of serum LDH has been verified (21,31,35-38). Similarly, in this study on the prognostic role of LDH in TC, we found that the elevated pretreatment serum LDH level was associated with poor OS and short DFS after resection of TC. It implied that management in patients with high LDH levels should be change. Multimodality therapy, such as neoadjuvant chemotherapy should be considered for these patients.

In addition, we found that the elevated pretreatment serum LDH level was associated with male patients and larger tumor size, which indicated that male patients and larger tumor size might predict the elevated pretreatment serum LDH level.

PORT is usually administrated for TC. However, the role of PORT in TC is still controversial. Due to the rarity of TC, no prospective studies that focused on PORT are available. There are only several large-sample retrospective studies on PORT. Studies from the European Society of Thoracic Surgeons (ESTS) and the Chinese Alliance for Research of Thymoma (ChART) showed that PORT improved OS of TC $(4,18)$. However, studies from the International Thymic Malignancy Interest Group (ITMIG) and the Japanese Association for Research on the Thymus (JART) showed PORT improved DFS but not OS for TC $(7,17)$. The present analysis also showed that PORT improved only DFS but not OS rate of the patients.

Bott et al. note that treatment of patients with recurrent or progressive thymic tumors is associated with long-term survival (39). In the present study, the 1-, and 3-year survival after recurrence were $53.8 \%$, and $37.8 \%$, respectively. It indicated that treatments after recurrence such as surgical resection were still effective for patients with TC.

Completeness of resection is an important prognostic factor of TC. Almost all reports on surgical treatment of $\mathrm{TC}$ indicate that complete resection is an independent prognostic factor in patients with TC $(4,7,17,18)$. In this study, R0 resection was not associated with OS. The possible explanation was effective PORT and treatments after recurrence.

The major limitations of this study are small series and its retrospective nature. The reference range of $\mathrm{LDH}$ was different among different institution, and we used the values specified in our center.
To our knowledge, this is the first study to report pretreatment serum LDH level in relation to prognosis of TC patients treated with surgical resection. In this study, we found that elevated pretreatment serum LDH level was associated with inferior OS and DFS, and might have therapeutic implication for these patients. Since the study was small series, larger prospective study is necessary for verifying the prognostic role of pretreatment serum LDH level in TC in the future.

\section{Acknowledgements}

None.

\section{Footnote}

Conflicts of Interest: The authors have no conflicts of interest to declare.

Ethical Statement: All procedures performed in studies involving human participants were in accordance with the ethical standards of the institutional and/or national research committee and with the 1964 Helsinki declaration and its later amendments or comparable ethical standards. A waiver of informed consent was requested, and the approval was obtained from independent ethics committees at Sun Yat-Sen University Cancer Center (number of the ethic approval: B2017-041).

\section{References}

1. Greene MA, Malias MA. Aggressive multimodality treatment of invasive thymic carcinoma. J Thorac Cardiovasc Surg 2003;125:434-6.

2. Omasa M, Date H, Sozu T, et al. Postoperative radiotherapy is effective for thymic carcinoma but not for thymoma in stage II and III thymic epithelial tumors: the Japanese Association for Research on the Thymus Database Study. Cancer 2015;121:1008-16.

3. Weissferdt A, Moran CA. Thymic carcinoma, part 1: a clinicopathologic and immunohistochemical study of 65 cases. Am J Clin Pathol 2012;138:103-14.

4. Ruffini E, Detterbeck F, Van Raemdonck D, et al. Thymic carcinoma: a cohort study of patients from the European society of thoracic surgeons database. J Thorac Oncol 2014;9:541-8.

5. Margaritora S, Cesario A, Cusumano G, et al. Thirty-fiveyear follow-up analysis of clinical and pathologic outcomes 
of thymoma surgery. Ann Thorac Surg 2010;89:245-52; discussion 252.

6. Shintani Y, Inoue M, Kawamura T, et al. Multimodality treatment for advanced thymic carcinoma: outcomes of induction therapy followed by surgical resection in 16 cases at a single institution. Gen Thorac Cardiovasc Surg 2015;63:159-63.

7. Ahmad U, Yao X, Detterbeck F, et al. Thymic carcinoma outcomes and prognosis: results of an international analysis. J Thorac Cardiovasc Surg 2015;149:95-100, 101.e1-2.

8. Wang S, Wang Z, Liu X, et al. Prognostic factors of patients with thymic carcinoma after surgery: a retrospective analysis of 58 cases. World J Surg 2014;38:2032-8.

9. Song Z, Zhang Y. Adjuvant therapy in stage II thymic carcinoma. J Cancer Res Clin Oncol 2014;140:349-52.

10. Filosso PL, Guerrera F, Rendina AE, et al. Outcome of surgically resected thymic carcinoma: a multicenter experience. Lung Cancer 2014;83:205-10.

11. Zhao Y, Zhao H, Hu D, et al. Surgical treatment and prognosis of thymic squamous cell carcinoma: a retrospective analysis of 105 cases. Ann Thorac Surg 2013;96:1019-24.

12. Weksler B, Dhupar R, Parikh V, et al. Thymic carcinoma: a multivariate analysis of factors predictive of survival in 290 patients. Ann Thorac Surg 2013;95:299-303.

13. Hosaka Y, Tsuchida M, Toyabe S, et al. Masaoka stage and histologic grade predict prognosis in patients with thymic carcinoma. Ann Thorac Surg 2010;89:912-7.

14. Cardillo G, Carleo F, Giunti R, et al. Predictors of survival in patients with locally advanced thymoma and thymic carcinoma (Masaoka stages III and IVa). Eur J Cardiothorac Surg 2010;37:819-23.

15. Lee CY, Bae MK, Park IK, et al. Early Masaoka stage and complete resection is important for prognosis of thymic carcinoma: a 20-year experience at a single institution. Eur J Cardiothorac Surg 2009;36:159-62; discussion 163.

16. Ogawa K, Toita T, Uno T, et al. Treatment and prognosis of thymic carcinoma: a retrospective analysis of 40 cases. Cancer 2002;94:3115-9.

17. Hishida T, Nomura S, Yano M, et al. Long-term outcome and prognostic factors of surgically treated thymic carcinoma: results of 306 cases from a Japanese Nationwide Database Study. Eur J Cardiothorac Surg 2016;49:835-41.

18. Fu H, Gu ZT, Fang WT, et al. Long-Term Survival After Surgical Treatment of Thymic Carcinoma: A Retrospective Analysis from the Chinese Alliance for Research of
Thymoma Database. Ann Surg Oncol 2016;23:619-25.

19. Walenta S, Mueller-Klieser WF. Lactate: mirror and motor of tumor malignancy. Semin Radiat Oncol 2004;14:267-74.

20. Quoix E, Purohit A, Faller-Beau M, et al. Comparative prognostic value of lactate dehydrogenase and neuronspecific enolase in small-cell lung cancer patients treated with platinum-based chemotherapy. Lung Cancer 2000;30:127-34.

21. Zhou GQ, Tang LL, Mao YP, et al. Baseline serum lactate dehydrogenase levels for patients treated with intensitymodulated radiotherapy for nasopharyngeal carcinoma: a predictor of poor prognosis and subsequent liver metastasis. Int J Radiat Oncol Biol Phys 2012;82:e359-65.

22. Shamash J, Oliver RT, Gallagher CJ, et al. Pre-induction $\mathrm{LDH}$ as a prognostic factor for outcome of high dose chemotherapy (HDCT) for germ cell tumours relapsing or refractory to conventional chemotherapy. Br J Cancer 2000;82:2022-3.

23. Camp RL, Dolled-Filhart M, Rimm DL. X-tile: a new bio-informatics tool for biomarker assessment and outcome-based cut-point optimization. Clin Cancer Res 2004;10:7252-9.

24. Marx A, Strobel P, Badve SS, et al. ITMIG consensus statement on the use of the WHO histological classification of thymoma and thymic carcinoma: refined definitions, histological criteria, and reporting. J Thorac Oncol 2014;9:596-611.

25. Scorsetti M, Leo F, Trama A, et al. Thymoma and thymic carcinomas. Crit Rev Oncol Hematol 2016;99:332-50.

26. Weissferdt A, Moran CA. Staging of thymic epithelial neoplasms: thymoma and thymic carcinoma. Pathol Res Pract 2015;211:2-11.

27. Huang J, Rizk NP, Travis WD, et al. Comparison of patterns of relapse in thymic carcinoma and thymoma. J Thorac Cardiovasc Surg 2009;138:26-31.

28. Masaoka A. Staging system of thymoma. J Thorac Oncol 2010;5:S304-12.

29. Blumberg D, Burt ME, Bains MS, et al. Thymic carcinoma: current staging does not predict prognosis. J Thorac Cardiovasc Surg 1998;115:303-8; discussion 308-9.

30. Ou W, Li N, Wang SY, et al. Phase 2 trial of neoadjuvant bevacizumab plus pemetrexed and carboplatin in patients with unresectable stage III lung adenocarcinoma (GASTO 1001). Cancer 2016;122:740-7.

31. Zhang SS, Xie X, Wen J, et al. TRPV6 plays a new role in predicting survival of patients with esophageal squamous cell carcinoma. Diagn Pathol 2016;11:14. 
32. Quist J, Hill AR. Serum lactate dehydrogenase (LDH) in Pneumocystis carinii pneumonia, tuberculosis, and bacterial pneumonia. Chest 1995;108:415-8.

33. Rademakers SE, Lok J, van der Kogel AJ, et al. Metabolic markers in relation to hypoxia; staining patterns and colocalization of pimonidazole, HIF-1alpha, CAIX, LDH-5, GLUT-1, MCT1 and MCT4. BMC Cancer 2011;11:167.

34. Gatenby RA, Gillies RJ. Why do cancers have high aerobic glycolysis? Nat Rev Cancer 2004;4:891-9.

35. Wan XB, Wei L, Li H, et al. High pretreatment serum lactate dehydrogenase level correlates with disease relapse and predicts an inferior outcome in locally advanced nasopharyngeal carcinoma. Eur J Cancer 2013;49:2356-64.

36. Wei Z, Zeng X, Xu J, et al. Prognostic value of pretreatment serum levels of lactate dehydrogenase in

Cite this article as: Liu Q, Fu X, Su X, Wang X, Zhang Y, Yang H, Hu Y, Wen J, Fu J. Elevated pretreatment serum lactate dehydrogenase level predicts inferior overall survival and disease-free survival after resection of thymic carcinoma. J Thorac Dis 2017;9(11):4550-4560. doi: 10.21037/ jtd.2017.10.86 nonmetastatic nasopharyngeal carcinoma: single-site analysis of 601 patients in a highly endemic area. Onco Targets Ther 2014;7:739-49.

37. Seki K, Tsuduki Y, Ioroi T, et al. Serum lactate dehydrogenase levels as a predictive marker of oxaliplatininduced hypersensitivity reactions in Japanese patients with advanced colorectal cancer. Int J Med Sci 2014;11:641-5.

38. Hui EP, Chan AT, Pezzella F, et al. Coexpression of hypoxia-inducible factors 1 alpha and 2 alpha, carbonic anhydrase IX, and vascular endothelial growth factor in nasopharyngeal carcinoma and relationship to survival. Clin Cancer Res 2002;8:2595-604.

39. Bott MJ, Wang H, Travis W, et al. Management and outcomes of relapse after treatment for thymoma and thymic carcinoma. Ann Thorac Surg 2011;92:1984-91; discussion 1991-2. 